\title{
Tunnelling measurements as a new method of investigation of thin film superconducting cuprate junctions
}

\author{
B. Chesca \\ Department of Physics, Loughborough University, UK
}

\begin{abstract}
Tunnelling measurements in thin film superconducting junctions involving cuprates (like $\mathrm{YBa}_{2} \mathrm{Cu}_{3} \mathrm{O}_{7}$ or $\mathrm{La}_{2-\mathrm{x}} \mathrm{Ce}_{\mathrm{x}} \mathrm{CuO}_{4-\mathrm{y}}$ ) are emerging as a unique tool of investigation. Thus, looking into tunnelling of Cooper pairs (the current that flows with no resistance) or quasiparticles in specially designed junctions is the most reliable method to investigate the symmetry of the superconducting order parameter in cuprates. This is crucial to establish a pairing mechanism for the electrons, i.e., the formation of Cooper pairs in these materials - one of the most important unsolved problems in modern solid state physics. Then, a detailed investigation of both dc and ac tunnelling of Cooper pairs in these junctions is essential to determine the current-phase relation (the relation between the current and the phase of the superconducting condensate). This represents a key element required in many applications like low-dissipative superconducting electronics or superconducting qubits in quantum computation. Also, tunnelling of quasiparticles in these junctions represent an accurate new method to determine the second critical field of cuprates. Here an overview on all these fundamental and applied aspects of this interesting class of nano materials will be given.
\end{abstract}

Keywords: unconventional superconductivity, thin film superconducting cuprate junctions, low temperature electric transport measurements.

\section{Introduction}

Understanding the mechanism responsible for superconductivity in unconventional superconductors, in particular in the high transition temperature superconductors (HTS), i.e., the cuprates discovered in 1986 [1] has been one of 
the major goals of condensed-matter physicists. What we do know about these materials is that the mechanism for superconductivity is fundamentally different than for conventional BCS [2] superconductors. Essential to the successful development of a microscopic theory for unconventional superconductors is the knowledge of the symmetry of the superconducting order parameter (SOP) or condensate wave function that describes the pairing of electrons in the superconductive state. By unconventional, we mean a state with an SOP that has a symmetry in momentum space different from that of the isotropic $s$-wave Cooper pair state that is believed to describe the conventional superconductors. Much of the recent attention has been focused on particular states with $\mathrm{d}_{\mathrm{x}}{ }^{2}-\mathrm{y}(d)$ wave [3], $\mathrm{d}_{\mathrm{xy}}$-wave, $p$-wave symmetry and also imaginary combinations like $s+i d, d+i \mathrm{~d}_{\mathrm{xy}}$ [4] all being highly anisotropic and characterized by a sign change in the SOP. These types of symmetry are implied by a number of possible superconducting pairing mechanisms, particularly those involving magnetic interactions that are known to be important in the cuprates.

Whereas for hole doped cuprates the $d$-wave symmetry has been established, for electron doped cuprates $R_{2-\mathrm{x}} \mathrm{Ce}_{\mathrm{x}} \mathrm{CuO}_{4}(R \mathrm{CCO}$ with $R=\mathrm{La}, \mathrm{Nd}, \mathrm{Pr}, \mathrm{Sm} \mathrm{Eu})$ where carriers are predominantly electrons the issue remains controversial. Another important issue is the determination of the SOP in other recently discovered presumably unconventional superconductors. One example is the natural superconductor/ferromagnet hybride $\mathrm{RuSr}_{2} \mathrm{GdCu}_{2} \mathrm{O}_{8}$, an exotic superconductor exhibiting the coexistence of superconductivity and ferromagnetism. The search for unconventional superconductivity received a significant boost from recent phase-sensitive experiments [5] that established $p$ wave symmetry in the $\mathrm{Sr}_{2} \mathrm{RuO}_{4}$ compound. In addition there is strong evidence that plutonium compound $\mathrm{PuCoGa}_{5}$ [6] as well as heavy fermions $\mathrm{CeRhIn}_{5}$ [7] are also unconventional superconductors. Thus, unconventional superconductivity appears to be a much more wide spread phenomenon among superconductors having very different crystal structure, as well as, chemical and physical properties, as previously believed. It should therefore not any more be exclusively related to the $\mathrm{CuO}$ planes that characterizes the structure of cuprates, but, to a somewhat more general common feature of various superconductors that has to be identified and understood. That appears to be one of the most challenging unsolved problems in condensed matter physics.

In this paper, I will review some of the most recent developments in the field of unconventional superconductivity in cuprates. Several topics will be covered:

section 2: the SOP in electron doped cuprates;

section 3: the current-phase relation in hole doped cuprate junctions;

section 4: the upper critical field in electron doped cuprates, i.e., the value of the applied external magnetic field that completely suppresses superconductivity.

\section{Unconventional superconductivity in cuprates}

There are several well established methods to determine the SOP in unconventional superconductors. Here we concentrate on one of the most powerful ones. It consists of looking into unconventional SOP-induced 
anomalies in the electric transport measurements of superconducting interferometers. The basic concept of such experiments is to create an interferometer consisting of a closed superconducting ring incorporating two Josephson junctions connected in parallel. The interferometer is specially designed to probe the phase of the pair wave function in specific directions of interest. To test the pairing symmetry of the SOP of an electron doped cuprate, namely, the $\mathrm{La}_{2-\mathrm{x}} \mathrm{Ce}_{\mathrm{x}} \mathrm{CuO}_{4-\mathrm{y}}$, in [8], such an interferometer has been fabricated and its electric transport properties have been measured. In particular, the Josephson junction critical current $I_{c}$ as a function of a magnetic field B applied perpendicular to the planar thin film interferometers (see Fig. 1) has been measured. $I_{c}$ is defined as the maximum value of the supercurrent in a Josephson junction, i.e., the maximum current that flows with no resistance. The interferometers were constructed as follow. A $0.5 \mu \mathrm{m}$ thick c-axis oriented $\mathrm{La}_{2}$ ${ }_{x} \mathrm{Ce}_{\mathrm{x}} \mathrm{CuO}_{4-\mathrm{y}}$ thin film was epitaxially grown on a tetracrystal $\mathrm{SrTiO}_{3}$ substrate by molecular-beam epitaxy. The film was near optimal doping with $\mathrm{x}=0.105$ and $\mathrm{a}$ critical temperature of about $\mathrm{T}_{\mathrm{c}}=29 \mathrm{~K}$. The tetracrystal $\mathrm{SrTiO}_{3}$ substrate contains three identical $30^{\circ}$ [001] tilt symmetric grain boundaries (denoted by 1 , 2 , and 3 in Fig. 1(a)) that make an angle of $120^{\circ}$ with respect to each other. Grain boundary 3 is not optically visible so that it has been represented by red dotted line in Fig. 1(a). Subsequently the film was patterned by standard photolithography and Ar ion milling to form three interferometers photographed in Fig. 1(a). All three interferometers have two junctions of identical width 500 $\mu \mathrm{m}$ and a rectangular hole of width $160 \mu \mathrm{m}$ and height $300 \mu \mathrm{m}$. The hole of the central interferometer contains the "tetracrystal point". The junctions of this interferometer are located at the grain boundaries labelled as " 1 " and " 2 ". In the measurements the interferometers are biased with a dc current $I$ and the $d c$ voltage $U$ across the two junctions is recorded. The current has been swept from 0 up to a maximum positive value of about $10 \mu \mathrm{A}$, then back to 0 , then down to a minimum negative value of about $-10 \mu \mathrm{A}$, and finally back up to 0 again. A family of several hundreds such $I(U)$ sweeps each recorded for different B values between $-0.8 \mu \mathrm{T}$ and $0.8 \mu \mathrm{T}$ were then used to extract the $I_{c}(B)$ characteristic. An anomalous $I_{c}(B)$ pattern with a central minimum has been measured for the central interferometer, called $\pi$-design. This highly unusual result is in high contrast with a Fraunhoffer-like pattern, i.e., an $I_{c}(B)$ curve with a central maximum, that has been obtained for the other two interferometers that are conventional (i.e., they contain no $\pi$-junctions). Such a difference can only be explained if assuming that the superconducting order parameter in the optimally doped $\mathrm{La}_{2-\mathrm{x}} \mathrm{Ce}_{\mathrm{x}} \mathrm{CuO}_{4-\mathrm{y}}$ has a predominantly $d$-wave symmetry schematically represented in Fig. 1(a) with red and white lobes. Another important implication of such an anomalous $I_{c}(B)$ is that the interferometer measured consists of a socalled $\pi$-junction (i.e., a junction with a negative critical current $I_{c}$ ) connected in parallel to a conventional junction (i.e., a junction with a positive critical current $I_{c}$ ). Such $\pi$-junctions are ideal for implementation of complementary low dissipative superconducting digital electronics [9]. 


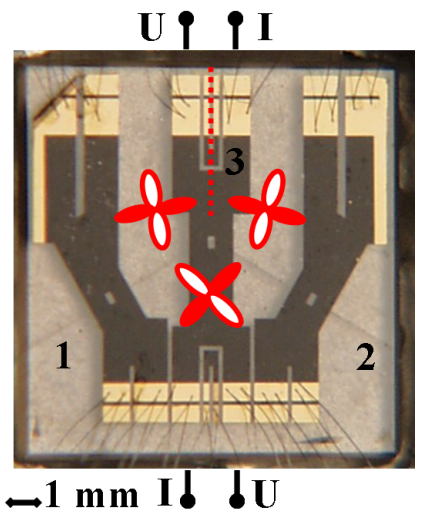

(a)

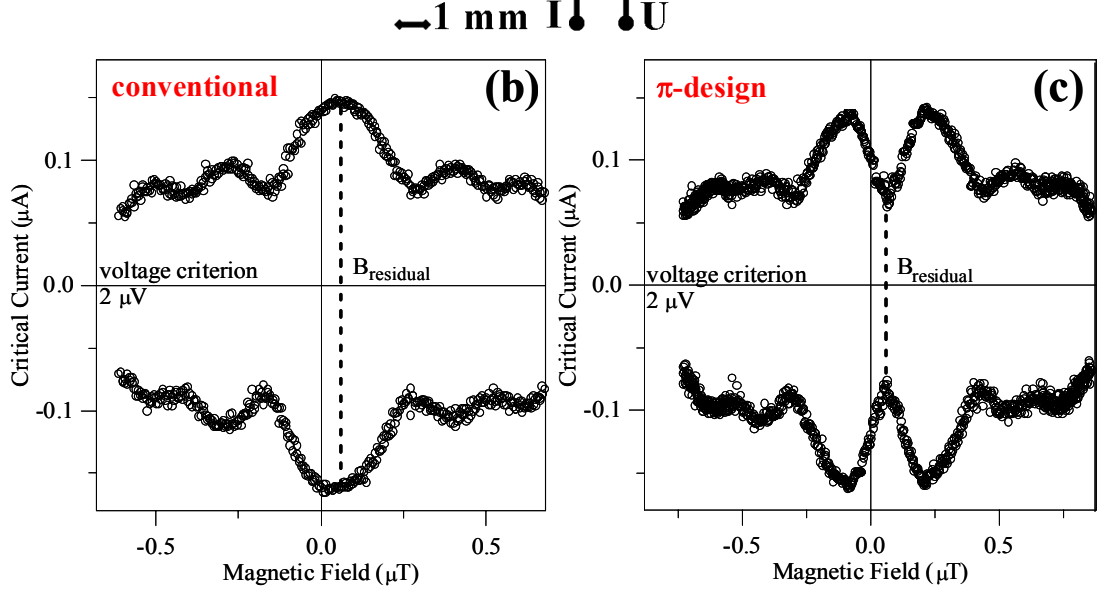

Figure 1: $\quad$ First realization of a $\pi$-junction (having a negative Josephson critical current) made of an electron doped cuprate. (a) the photograph of the three interferometers patterned. (b) The two side interferometers have a conventional Fraunhoffer-like $I_{c}(B)$ pattern. (c) The central interferometer $(\pi$-design) consisting of a $\pi$-junction in parallel to a conventional one has an anomalous $I_{c}(B)$ with a central minimum.

\section{Current-phase relation of thin film cuprate Josephson junctions}

Josephson junctions formed between two superconductors of which at least one is an unconventional one, or so called, a $d$-wave superconductor are very attractive candidates for the implementation of superconducting qubits in quantum computation [10] or $\pi$-junctions in Josephson (low-dissipative) digital circuits [9]. In addition, arrays of such $d$-wave junctions are of interest as model systems for studying magnetic phenomena-including frustration effects - in Ising antiferromagnets [11]. Moreover, $d$-wave junctions are among the most 
reliable tools to investigate the unconventional SOP in these materials [3]. The physics of $d$-wave junctions, however, is not fully understood. A key element, namely the knowledge of the current-phase relation (CPR) of the Josephson current remains unsettled [12]. It has been predicted [13-17] that zero-energy Andreev states (ZES) formed at the $d$-wave junctions interface are expected to induce a second harmonic Josephson current $J_{2}$ in the CPR. For various qubit concepts this $J_{2}$ is essential, as a superconducting qubit based on $J_{2}$ will have an operating point intrinsically stable and protected against the environmental noise, which will reduce decoherence [18]. Whereas it is now well understood that $d$ wave induces formation of ZES states [3] the existence of $J_{2}$ has been an intriguing unconfirmed prediction for a long time. Recently, this issue has been addressed [19] for Josephson junctions made between the $d$-wave $\mathrm{YBa}_{2} \mathrm{Cu}_{3} \mathrm{O}_{7-\mathrm{x}}$ and the conventional s-wave $\mathrm{Nb}$. There the authors first provided evidence for the formation of ZES and, secondly they searched for the existence of $J_{2}$. If it exists, this second harmonic component is expected [13-17] to be highly anisotropic as we change the tunneling orientation in the $a b$ plane reaching its maximum for tunneling close to [110] direction and its minimum for the [100] or [010] directions.

$J_{2}$ is expected [7-11] to produce a deviation from the standard sinusoidal CPR $\left(J_{c}(\varphi)=J c_{1} \sin (\varphi)\right)$ of the Josephson current density $J_{c}[6]$

$$
J_{c}(\varphi)=J_{1}+J_{2}=J c_{1} \sin (\varphi)+J c_{2} \sin (2 \varphi)
$$

Here $\varphi$ is the phase difference across the junction. For a purely $d$-wave order parameter as we increase $\theta$ (the angle in the $a b$ plane between the normal to the junction interface and the [100] crystal axis) starting from $0, J_{2}$ is expected to increase monotonically up to $\theta=45^{\circ}$ which corresponds to tunneling into the [110] direction. It should then decrease monotonically as we further increase $\theta$ from $45^{\circ}$ to $90^{\circ}$, corresponding to tunneling into the [010] direction. In particular, for tunneling close to the [110] direction, where $J_{l}$ vanishes due to the nodes of the $d$-wave order parameter, $J_{2}$ will dominate the CPR.

In [19] the authors prepared thin film ramp-edge junctions between 170-nm untwinned $\mathrm{YBa}_{2} \mathrm{Cu}_{3} \mathrm{O}_{7-\mathrm{x}}$ and $150-\mathrm{nm} \mathrm{Nb}$ using a 30-nm Au barrier. The use of untwinned $\mathrm{YBa}_{2} \mathrm{Cu}_{3} \mathrm{O}_{7-\mathrm{x}}$ thin films is especially important because otherwise $J_{2}$ may be strongly suppressed due to excessive diffusive scattering at the twin boundaries. Also, $J_{2}$ may be averaged out for a badly defined nodal orientation in a twinned film. The junctions are fabricated on the same chip, and the angle $\theta$ with the $\mathrm{YBa}_{2} \mathrm{Cu}_{3} \mathrm{O}_{7-\mathrm{x}}$ crystal $b$-axis is varied in units of 5 degrees, so that tunnelling can be probed in $360^{\circ} / 5^{\circ}=72$ different directions in the $a b$ plane (see Fig. 2). The growth of untwinned $\mathrm{YBa}_{2} \mathrm{Cu}_{3} \mathrm{O}_{7-\mathrm{x}}$ films [20], as well as detailed order parameter issues [21], and ZES-assisted quasiparticle tunneling [22] in these particular junctions were reported elsewhere. All 72 junctions were $4 \mu \mathrm{m}$ wide. Quasiparticle conductance spectra $G(V)$ of all 72 junctions were measured for a wide range of temperatures $\mathrm{T}(4.2-77 \mathrm{~K})$ and magnetic fields $B(0-7 \mathrm{~T})$. It was found that all observed features, in particular a well defined zero-bias conductance peak (ZBCP), were consistent with a convolution of density of states with broadened ZES formed at the $\mathrm{YBa}_{2} \mathrm{Cu}_{3} \mathrm{O}_{7-\mathrm{x}} / \mathrm{Au} / \mathrm{Nb}$ junction interfaces [19]. Here a summary will be given of some of the most important 
findings from a qualitative point of view. The authors observed the same qualitative picture independent of the tunneling direction (see Fig. 3). At $4.2 \mathrm{~K}$ and a small $B$ of $0.01 \mathrm{~T}$, which is large enough to completely suppress the $d c$ Josephson current well-defined $\mathrm{Nb}$ coherence peaks and a dip at the center of a broadened zero-bias conductance peak (ZBCP) were observed. As superconductivity is suppressed in $\mathrm{Nb}$, by increasing $T$ from $4.2 \mathrm{~K}$ up to slightly below the critical temperature of $\mathrm{Nb}\left(T_{\mathrm{c}, \mathrm{Nb}} \approx 9.1 \mathrm{~K}\right)$ or $B$ from $0.1 \mathrm{~T}$ up to slightly below the second critical field of $\mathrm{Nb}\left(B_{\mathrm{c} 2, \mathrm{Nb}} \approx 1.15 \mathrm{~T}\right)$ the $\mathrm{Nb}$ coherence peaks become suppressed and the ZBCP-presence gradually manifests. Close to the critical temperature $T_{\mathrm{c}, \mathrm{Nb}}$ (see Fig. 3(a)) or to $0.4 \mathrm{~T}$ (see Fig. 3(b)) no trace is left of the $\mathrm{Nb}$ coherence peaks, while the ZBCP is fully developed. That provides clear evidence for the formation of ZES. Increasing $T$ or $B$ even further (from $T_{\mathrm{c}}$, $\mathrm{Nb}$ up to $77 \mathrm{~K}$, or $B$ from $0.4 \mathrm{~T}$ to $B_{\mathrm{c} 2, \mathrm{Nb}}$ and further to $7 \mathrm{~T}$ ), however, a significant difference appears between the $T$ and $B$ dependence of $G(V)$. The ZBCP (its amplitude and width) is essentially not affected by an increase of $B$, while by increasing $T$ the ZBCP becomes strongly suppressed and widens. In particular, we could not observe any trace of a ZBCP at $77 \mathrm{~K}$. The remarkable insensitivity of $G(V)$ to the tunneling direction strongly suggests the existence of ZES in all tunneling orientations in the $a b$ plane, including the [100] and [010] directions. The authors believe this is a signature of diffusive reflection or scattering, possibly due to microscopic interface roughness.

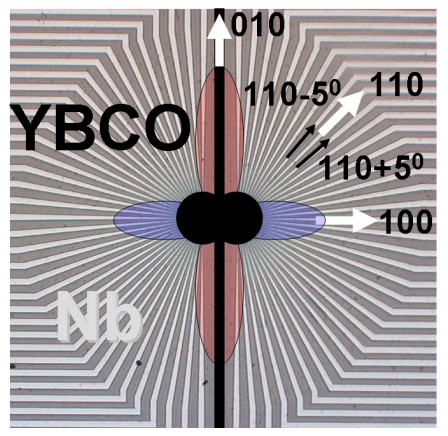

Figure 2: Topview photograph of the untwinned $\mathrm{YBa}_{2} \mathrm{Cu}_{3} \mathrm{O}_{7-\mathrm{x}} / \mathrm{Au} / \mathrm{Nb}$ ramptype junction layout. The YBCO base electrode (in black) is contacted by a Au barrier (not shown) and a $\mathrm{Nb}$ counterelectrode (light-gray). Tunneling is tested in 72 different directions in the $a b$ plane. The arrows indicate some of those tunneling directions.

Now that there is solid evidence of ZES formation, then what about $J_{2}$ : does it exists, can it be measured? A powerful experiment on $J_{2}$ concerns Shapiro steps. It is well known that if the CPR is purely sinusoidal $\left(J_{c_{2}}=0\right.$ in Eq. (1)) microwave radiation of frequency $f$ will induce Shapiro steps at integer $n$ multiples of the voltage $\mathrm{V}_{0}$, satisfying the Josephson voltage-frequency relation 

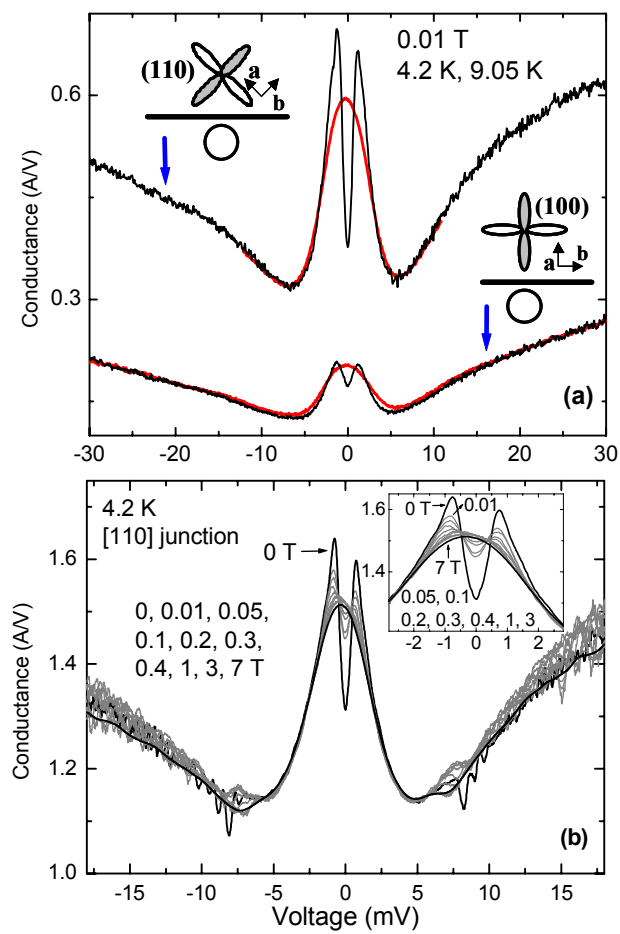

Figure 3: (a) Conductance spectra of 2 junctions with different tunneling directions (100 and 110) at $4.2 \mathrm{~K}$ (in red) and just below $\mathrm{T}_{\mathrm{c}, \mathrm{Nb}}$. Insets show schematically the isotropic s-wave SOP in $\mathrm{Nb}$ and the highly anisotropic d-wave SOP in the cuprate, as well as, the orientation of the $a$ and $b$ axis of the unit cell. (b) Conductance spectra of a [110]-oriented junction for ten different magnetic field values from $0 \mathrm{~T}$ (in black) up to $7 \mathrm{~T}$ (in black). The inset shows details of the low voltage spectra.

$f / \mathrm{V}_{0}=0.486 \mathrm{GHz} / \mu \mathrm{V}$. If $J c_{2}$ is finite also half-integer Shapiro steps should appear at multiples of $\mathrm{V}_{0} / 2$ [23-25]. If half-integer Shapiro steps are not observed then the presence of a significant $J_{2}$ in the CPR can be ruled out. In [19] a very detailed search was performed in the entire frequency range where integer Shapiro steps could be observed. A carefully examination was done of every 10 $\mathrm{MHz}$ frequency interval within the 1-20 GHz region. Such an approach was repeated for all junctions investigated. Typical sets of current-voltage characteristics are shown in Figs.4(a)-4(c) for three junctions: [100], [110] and [110]-5 $5^{\circ}$. Well-defined integer Shapiro steps in accordance with the theoretical expectations are clearly visible. Pronounced integer Shapiro steps up to $n=21$ (as 

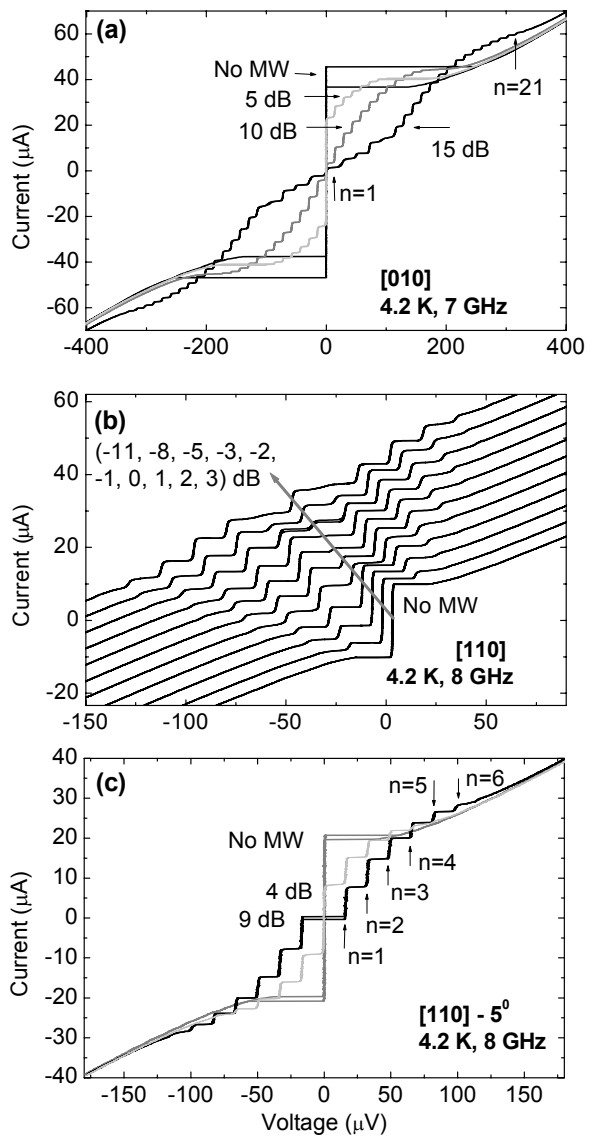

Figure 4: Integer Shapiro steps (indicated by vertical arrows) at $4.2 \mathrm{~K}$ of [010], [110], and [110]-5'-oriented junctions at different microwave amplitudes. For clarity, the current-voltage characteristics in (b) are shifted in diagonal direction shown by the gray line.

in Fig. 4(a)) or even higher in some cases were detected. However, no trace of half-integer Shapiro steps was found in any of the junctions, although particular attention was given to those microwave amplitudes where the integer Shapiro steps or the $I_{c}$ vanishes and consequently the half-integer Shapiro steps are expected to be most pronounced. These observations strongly suggest that $J_{2}$ in these junctions is so small (if present at all !) that it cannot be measured. In conclusion $J_{2}$ should be completely ignored in Eq.(1) so that the junctions investigated have to be considered as having a pure sinusoidal CPR. 


\section{Second critical field of thin film cuprate superconductors}

Mapping the magnetic field-temperature (B-T) phase diagram of cuprate superconductors is essential for their understanding. Unlike conventional type-II superconductors where the B-T phase diagram consists of the Meissner phase, the Shubnikov phase and the normal state, the phase diagram of cuprates superconductors is extremely complex, exhibiting a variety of vortex phases [26] and also an intriguing pseudogap region [27,28]. The transition between the superconducting state and the normal state, and thus the relation between the superconducting and the pseudogap states, is hard to determine, not only due to the large values of the second critical field $B_{c 2}$ in the case of hole-doped cuprates, but also because of the presence of vortex liquid phases as well as strong fluctuation effects, leading to nonzero resistance well below $B_{c 2}$. For the electron-doped cuprates $\operatorname{Pr}_{2}{ }_{x} \mathrm{Ce}_{x} \mathrm{CuO}_{4}$ and $\mathrm{Nd}_{2}{ }_{x} \mathrm{Ce}_{x} \mathrm{CuO}_{4}$ resistive measurements [29] or the vortex Nernst signal [30-32] revealed $B_{c 2}$ (at temperature $0 \mathrm{~K}$ ) values in the range of $7-10 \mathrm{~T}$. For $\mathrm{La}_{2}{ }_{x} \mathrm{Ce}_{x} \mathrm{CuO}_{4}$ an analysis of the vortex pinning strength yielded $B_{c 2}$ (at temperature $0 \mathrm{~K}$ ) of about $9 \mathrm{~T}$ [33]. However, the various methods applied to determine $B_{c 2}$ often yield inconsistent results; see, e.g., the discussion in [29]. In [34] the authors showed that an analysis of ZES causing a zero bias conductance peak (ZBCP) - see Fig. 5 - in the conductance spectra of cuprate grain boundary junctions (GBJs) yields a new lower bound for $B_{c 2}$ which is at least a factor of 2.5 above previous estimates. ZES result from the constructive interference of Andreev reflected electron- and hole-like quasiparticles [35]. If the quasiparticles experience a sign change of the superconducting order parameter upon reflection, ZES appear at the Fermi energy, giving rise to the ZBCP [36]. A ZBCP caused by ABS relies on the phase coherence of the elementary excitations above the Cooper pairing ground state. It thus should vanish when the phase coherence is lost, i.e., at the transition between the superconducting state and the normal state. Such ZBCPs thus allow us to determine $B_{c 2}$, or at least to give a reasonable lower bound. Indeed, in [34] it has been shown that in the electron-doped cuprate $\mathrm{La}_{2}{ }_{x} \mathrm{Ce}_{x} \mathrm{CuO}_{4}$ the superconducting state persists to substantially higher magnetic fields than reported previously. Thus, well defined ZBCP have been observed for B field direction both parallel to the $\mathrm{c}$-axis or perpendicular to it (i.e., parallel to the $a b$ plane) for a wide range of temperatures from $4.2 \mathrm{~K}$ close to the transition temperature from the superconducting state to the normal state [37]. Some typical ZBCP measurements are shown in Fig. 5. The integrated density of states DOS (i.e, the area of the ZBCP) normalized to its value at $0.1 \mathrm{~T}$ versus $\mathrm{H}$ is shown in the insets of Figs. 5(a) and 5(b). The observed nonlinear dependence strongly suggests that the measured ZBCP is due to the formation of ZES at the junction interface. The ZBCP persists for B fields as high as $16 \mathrm{~T}$ [34]. A zero temperature extrapolation suggests that $B_{c 2}$ (at temperature $0 \mathrm{~K}$ ) is at least $25 \mathrm{~T}$. Extending superconductivity to such high fields shrinks the region where a pseudogap phase may exist. Such a method allows the investigation of the $B-T$ phase diagram via ZES. It should be applicable to any superconductor where the 


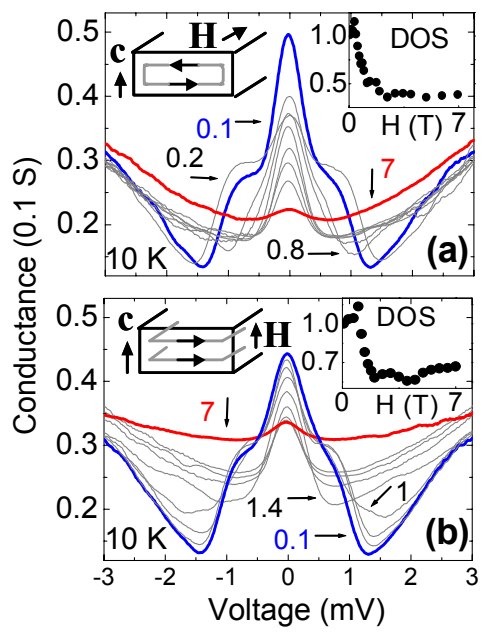

Figure 5: Variation of the G(V) spectra of a junction with magnetic field $H$ parallel to the $a b$ plane (a) or $\mathrm{H}$ parallel to the $c$-axis (b) at a temperature $T=10 \mathrm{~K}$. In the insets the junction cross section is shown schematically together with the direction of screening current flow (horizontal arrows). The unspecified values of $\mu_{0} \mathrm{H}$ are: (a) 1, 2, 2.5, $3 \mathrm{~T}$ (b) 0.2, 0.8, 2, 3, $4 \mathrm{~T}$; the numbers labeling the $\mathrm{G}(\mathrm{V})$ curves are field values in Tesla. In the insets the integrated density of states DOS normalized to its value at $0.1 \mathrm{~T}$ versus $\mathrm{H}$ are shown.

SOP changes sign (like for d-wave superconductivity), providing an effective additional tool to explore the superconducting state.

\section{References}

[1] Bednorz, J. G. \& Müller, K.A., Possible high $T_{\mathrm{c}}$ superconductivity in the $\mathrm{Ba}-\mathrm{La}-\mathrm{Cu}-\mathrm{O}$ system. Z. Phys. B 64, pp. 189-193, 1986.

[2] Bardeen, J., Cooper, L.N. \& Schrieffer, J.R., Theory of Superconductivity. Phys. Rev. 108, pp.1175-1204, 1957.

[3] Tsuei, C.C. \& Kirtley, J. R., Pairing symmetry in cuprate superconductors. Rev. Mod. Phys. 72, pp. 969-1016, 2000.

[4] Amin, M.H.S., Rashkeev, S.N., Coury, M., Omelyanchouk, A.N. \& Zagoskin, A.M., $d+i$ s versus $d+i d$ time reversal symmetry breaking states in finite size systems. Phys. Rev. B 66, pp. 174515, 2002.

[5] Nelson, K.D., Mao, Z.Q., Maeno, Y. \& Liu, Y., Odd-Parity Superconductivity in $\mathrm{Sr}_{2} \mathrm{RuO}_{4}$. Science 306, pp. 1151-1154, 2004. 
[6] Curro, N. J., Caldwell, T., Bauer, E. D., Morales, L. A., Graf, M. J., Bang, Y., Balatsky A. V., Thompson, J. D. \& Sarrao, J. L., Unconventional superconductivity in PuCoGa5. Nature 434, pp. 622-625, 2005.

[7] Park, T., Ronning, F., Yuan, H. Q., Salamon, M. B., Movshovich, R., Sarrao, J. L. \& Thompson, J. D., Hidden magnetism and quantum criticality in the heavy fermion superconductor CeRhIn5. Nature 440, pp. 65-68, 2006.

[8] Chesca, B., Ehrhardt, K., Mößle, M., Straub, R., Koelle, D., Kleiner, R. \& Tsukada, A., Magnetic-Field Dependence of the Maximum Supercurrent of $\mathrm{La}_{2-x} \mathrm{Ce}_{x} \mathrm{CuO}_{4-y}$ Interferometers: Evidence for a Predominant $d_{x-y}^{22}$ Superconducting Order Parameter. Phys. Rev. Lett, 90, pp. 057004, 2003.

[9] Ortlepp, T., Ariando, Mielke, O., Verwijs, C. J. M., Foo, K. F. K., Rogalla, H., Uhlmann, F. H. \& Hilgenkamp, H., Flip-Flopping Fractional Flux Quanta. Science 312, pp. 1495-1497, 2006.

[10] Ioffe, L. B., Geshkenbein, V. B., Feigel'man, M. V., Fauchere, A. L. \& Blatter, G., Environmentally decoupled sds -wave Josephson junctions for quantum computing. Nature 398, pp. 679-681, 1999.

[11] Hilgenkamp, H., Ariando, Smilde, H. J. H., Blank, D. H. A. , Rijnders, G., Rogalla, H., Kirtley, J. R. \& Tsuei, C. C., Ordering and manipulation of the magnetic moments in large-scale superconducting $\pi$-loop arrays. Nature 422, pp. 50-53, 2003.

[12] Golubov, A. A., Kupriyanov, M. Yu. \& Il'ichev, E., The current-phase relation in Josephson junctions. Rev. Mod. Phys. 76, pp. 411-469, 2004.

[13] Tanaka, Y. \& Kashiwaya, S., Theory of the Josephson effect in $d$-wave superconductors. Phys. Rev. B 53, pp. R11957-R11960, 1996.

[14] Barash, Yu., Burkhardt, S. H. \& Rainer, D., Low-Temperature Anomaly in the Josephson Critical Current of Junctions in $d$-Wave Superconductors. Phys. Rev. Lett. 77, pp. 4070-4073, 1996.

[15] Samanta, M. \& Datta, S., Effect of midgap states in d-wave superconductors on the flux quantization in tricrystal rings. Phys. Rev. B 55, pp. R8689-R8692, 1997.

[16] Tanaka, Y. \& Kashiwaya, S., Theory of Josephson effects in anisotropic superconductors. Phys. Rev. B 56, pp. 892-912, 1997.

[17] Riedel, R. A. \& Bagwell, P. F., Low-temperature Josephson current peak in junctions with $d$-wave order parameters. Phys. Rev. B 57, pp. 6084-6089, 1998.

[18] Amin, M. H. S., Smirnov, A. Yu., Zagoskin, A. M., Lindström, T., Charlebois, S. A., Claeson, T. \& Tzalenchuk, A. Ya., Silent phase qubit based on $d$-wave Josephson junctions. Phys. Rev. B 71, pp. 064516, 2005.

[19] Chesca, B., Smilde, H. J. H., \& Hilgenkamp, H., Upper bound on the Andreev states induced second harmonic in the Josephson coupling of $\mathrm{YBa}_{2} \mathrm{Cu}_{3} \mathrm{O}_{7-5} / \mathrm{Nb}$ junctions from experiment and numerical simulations. Phys. Rev. B, 77, pp. 184510, 2008.

[20] Dekkers, J.M., Rijnders, G., Harkema, S., Smilde, H. J. H., Hilgenkamp, H., Rogalla, H. \& Blank, D. H. A., Monocrystalline $\mathrm{YBa}_{2} \mathrm{Cu}_{3} \mathrm{O}_{7-x}$ thin films 
on vicinal $\mathrm{SrTiO}_{3}$ (001) substrates. Appl. Phys. Lett. 83, pp. 5199-5201, 2003.

[21] Smilde, H.J.H., Golubov, A. A., Ariando, Rijnders, G., Dekkers, J. M., Harkema, S., Blank, D. H., Rogalla, H. \& Hilgenkamp, H., Admixtures to $d$ - Wave Gap Symmetry in Untwinned $\mathrm{YBa}_{2} \mathrm{Cu}_{3} \mathrm{O}_{7}$ Superconducting Films Measured by Angle-Resolved Electron Tunneling. Phys. Rev. Lett. 95, pp. 257001, 2005.

[22] Chesca, B., Dönitz, D., Dahm, T., Huebener, R., Koelle, D., Kleiner, R., Ariando \& Hilgenkamp, H., Upper bound on the Andreev states induced second harmonic in the Josephson coupling of $\mathrm{YBa}_{2} \mathrm{Cu}_{3} \mathrm{O}_{7-\boldsymbol{s}} / \mathrm{Nb}$ junctions from experiment and numerical simulations. Phys. Rev. B 73, pp. 014529, 2006.

[23] Hasselberg, L.-E., Levinsen, M. T. \& Samuelsen, M. R., Subharmonic gap structure and subharmonic Josephson steps. J. Low Temp.Phys. 21, pp. 567$587,1975$.

[24] Gundlach, K. H. \& Kadlec, J., Current-versus voltage source model in Josephson effect. Phys. Lett. 63A, pp. 149-150, 1977.

[25] Kleiner, R., Katz, A. S., Sun, A. G., Summer, R., Gajewski, D. A., Han, S. H., Woods, S. I., Dantsker, E., Chen, B., Char, K., Maple, M. B., Dynes, R. C. \& Clarke, J., Pair Tunneling from $c$-Axis $\mathrm{YBa}_{2} \mathrm{Cu}_{3} \mathrm{O}_{7-x}$ to $\mathrm{Pb}$ : Evidence for $s$-Wave Component from Microwave Induced Steps. Phys. Rev. Lett. 76, pp. 2161-2164, 1996.

[26] Blatter, G., Feigel'man, M. V., Geshkenbein, V. B., Larkin, A. I. \& Vinokur, V. M., Vortices in high-temperature superconductors. Rev. Mod. Phys. 66, pp. 1125-1388, 1994.

[27] Damasscelli, A., Hussain, Z. \& Shen, Z. X., Angle-resolved photoemission studies of the cuprate superconductors. Rev. Mod. Phys. 75, pp. 473-541, 2003.

[28] Fisher, O., Kugler, M., Aprile, I. M. \& Berthod, C., Scanning tunneling spectroscopy of high-temperature superconductors. Rev. Mod. Phys. 79, pp. 353, 2007.

[29] Fournier, P. \& Greene, R. L., Doping dependence of the upper critical field of electron-doped $\mathrm{Pr}_{2-x} \mathrm{Ce}_{x} \mathrm{CuO}_{4}$ thin films. Phys. Rev. B 68, pp. 094507, 2003.

[30] Gollnik, F. \& Naito, M., Doping dependence of normal- and superconducting state transport properties of $\mathrm{Nd}_{2-x} \mathrm{Ce}_{x} \mathrm{CuO}_{4 \pm y}$ thin films. Phys. Rev. B 58, pp. 11734-11752, 1998.

[31] Balci, H., Hill, C. P., Qazilbash, M. M. \& Greene, R. L., Nernst effect in electron-doped $\operatorname{Pr}_{2-x} \mathrm{Ce}_{x} \mathrm{CuO}_{4}$. Phys. Rev. B 68, pp. 054520, 2003.

[32] Wang., Y., Ono, S., Onose, Y., Gu, G., Ando, Y., Tokura, Y., Uchida, S. \& Ong, N. P., Dependence of Upper Critical Field and Pairing Strength on Doping in Cuprates. Science 299, pp. 86-89, 2003.

[33] Zuev, Y., Lemberger, T. R., Skinta, J. A., Greibe, T., \& Naito, M., Vortex pinning in electron-doped cuprate superconductor $\mathrm{La}_{2-x} \mathrm{Ce}_{x} \mathrm{CuO}_{4}$. Phys. Status Solidi (b) 236, pp. 412-415, 2003. 
[34] Wagenknecht, M., Koelle, D., Kleiner, R., Graser, S., Schopohl, N., Chesca, B., Tsukada, A., Goennenwein, S. T., \& Gross, R., Phase Diagram of the Electron-Doped $\mathrm{La}_{2-\mathrm{x}} \mathrm{Ce}_{\mathrm{x}} \mathrm{CuO}_{4}$ Cuprate Superconductor from Andreev Bound States at Grain Boundary Junctions. Phys. Rev. Lett. 100, pp. 227001, 2008.

[35] Deutscher, G., Andreev-Saint-James reflections: A probe of cuprate superconductors. Rev. Mod. Phys. 77, pp. 109-135, 2005.

[36] Kashiwaya, S. \& Tanaka, Y., Tunnelling effects on surface bound states in unconventional superconductors. Rep. Prog. Phys. 63, pp. 1641-1724, 2000.

[37] Chesca, B., Seifried, M., Dahm, T., Schopohl, N., Koelle, D., Kleiner, R. \& Tsukada, A., Observation of Andreev bound states in bicrystal grain boundary Josephson junctions of the electron-doped superconductor $\mathrm{La}_{2-x} \mathrm{Ce}_{x} \mathrm{CuO}_{4-y}$. Phys. Rev. B 71, pp. 104504, 2005. 